\title{
PARABOLIC FACTORIZATIONS OF SPLIT CLASSICAL GROUPS
}

\author{
N. A. VAVILOV AND S. S. SINCHUK
}

To Andrei Suslin, with love and admiration

\begin{abstract}
An analog of the Dennis-Vaserstein decomposition is proved for an arbitrary pair of maximal parabolic subgroups $P_{r}$ and $P_{s}$ in split classical groups, under appropriate stability conditions. Before, such decompositions were only known for pairs of terminal parabolic subgroups.
\end{abstract}

\section{INTRODUCTION}

Let $\Phi=\mathrm{A}_{l}, \mathrm{~B}_{l}, \mathrm{C}_{l}, \mathrm{D}_{l}$ be a classical root system of rank $l$. Choose an order on $\Phi$ and let $\Phi^{+}, \Phi^{-}$, and $\Pi=\left\{\alpha_{1}, \ldots, \alpha_{l}\right\}$ be the corresponding sets of positive, negative, and fundamental roots, respectively. In the sequel all our notation pertaining to root systems, including the numbering of fundamental roots, follows that of [2].

Next, let $R$ be a commutative ring with 1 , and let $G(\Phi, R)$ be a Chevalley group of type $\Phi$ over $R$. Since the problems we consider are essentially independent of the weight lattice, in the sequel we usually construe $G(\Phi, R)$ as a certain split classical group, namely, $\mathrm{SL}(l+1, R)$ for type $\mathrm{A}_{l}, \mathrm{SO}(2 l+1, R)$ for type $\mathrm{B}_{l}, \operatorname{Sp}(2 l, R)$ for type $\mathrm{C}_{l}$, and finally, $\mathrm{SO}(2 l, R)$ for type $\mathrm{D}_{l}$. Here, the orthogonal groups always correspond to a form of the maximal Witt index.

In fact, usually we consider not the Chevalley group $G(\Phi, R)$ itself, but rather its elementary subgroup $E(\Phi, R)$ generated by the unipotent root subgroups $X_{\alpha}, \alpha \in \Phi$, elementary with respect to a split maximal torus $T(\Phi, R)$. For classical groups, this is one of the following groups: $E(l+1, R), \mathrm{EO}(2 l+1, R), \operatorname{Ep}(2 l, R), \operatorname{EO}(2 l, R)$; the definitions of these groups are recalled in $\S 2$.

We fix some $1 \leq s<r \leq l$ and let $P=\mathrm{EP}_{r}$ and $Q=\mathrm{EP}_{s}$ be the corresponding elementary maximal parabolic subgroups, whose definitions are recalled in $\S 3$. Most of the existing proofs of stability theorems for $K$-functors are based on decompositions of elementary subgroups $E(\Phi, R)$ - or of the groups $G(\Phi, R)$ themselves - in terms of pairs of terminal parabolic subgroups, for which $r, s=1, l$.

In the present paper we start a systematic study of similar decompositions for pairs of arbitrary, rather than terminal maximal parabolic subgroups. Our principal goal here is to prove the following result. It is interesting in itself, but it is aimed primarily at

2010 Mathematics Subject Classification. Primary 20G15, $20 \mathrm{G} 35$.

Key words and phrases. Split classical groups, elementary subgroup, parabolic subgroups, stability conditions, stable rank, unitary stable rank, Gauss decomposition, Bass-Kolster decomposition, DennisVaserstein decomposition.

The research of the first author was started in the framework of the RFFI project 08-01-00756 "Decompositions of algebraic groups and their applications in representation theory and $K$-theory". Apart from that, at the final stage his work was supported also by the RFFI projects 09-01-00762, 09-01-00784, 09-01-00878, 09-01-91333, 09-01-90304, and 10-01-90016. The second author acknowledges support of the RFFI project 10-01-92651 "Higher composition laws, algebraic $K$-theory and exceptional groups". 
applications to such problems as a description of various classes of subgroups, generation, and prestability.

The definitions of all relevant stability conditions are recalled in $\S 4$. When we talk of the unitary stable rank of a commutative ring $R$, it is viewed as a form ring with the pseudo-involution $h: x \mapsto-x$ and the form parameter $\Lambda=0$.

Theorem 1. Let $\mathrm{EP}_{r}$ and $\mathrm{EP}_{s}, 1 \leq s<r \leq l$, be two elementary maximal parabolic subgroups of the elementary Chevalley group $E(\Phi, R)$, and let $U_{r s}^{-}$be the intersection of the unipotent radicals of the opposite parabolic subgroups.

Assume that $\operatorname{sr}(R) \leq r-s$ and, moreover, that one of the following holds:

- $\Phi=\mathrm{A}_{l}, \mathrm{C}_{l}$;

- $\Phi=\mathrm{B}_{l}, \mathrm{D}_{l}, s \leq l-2$ and the condition $\Lambda S_{l-s-1}$ is fulfilled;

- $\Phi=\mathrm{D}_{l}, s=l-1, r=l$.

Then we have the decomposition

$$
E(\Phi, R)=\mathrm{EP}_{r} \cdot U_{r s}^{-} \cdot \mathrm{EP}_{s} .
$$

This theorem is well known in the following two extreme cases.

- Under the assumption $\operatorname{sr}(R)=1$ for $\Phi=\mathrm{A}_{l}, \mathrm{C}_{l}$ or $\operatorname{asr}(R)=1$ for $\Phi=\mathrm{B}_{l}, \mathrm{D}_{l}$. In this case, the claim follows from the Gauss decomposition, and even for $r=s$.

- In the case where $r=l, s=1$, our theorem boils down to the usual Dennis-Vaserstein decomposition. For type $\Phi=\mathrm{A}_{l}$, under the assumption $l \geq \operatorname{sr}(R)+1$ it was established in [16]. For other types, in a slightly weaker form, and under various stability conditions, it can be found in [10, 50, 42. Finally, in [19, 20] the genuine stability conditions were introduced.

- For type $\Phi=\mathrm{A}_{l}$, this theorem was proved in our paper [6].

The method that we use to prove this theorem in this paper allows us to establish, alongside with Theorem 1, its analog for the classical groups themselves.

Theorem 2. Let $P_{r}$ and $P_{s}, 1 \leq s<r \leq l$, be two maximal parabolic subgroups of the Chevalley group $G(\Phi, R)$.

Assume that $\operatorname{sr}(R) \leq r-s$ and, moreover, that one of the following holds:

- $\Phi=\mathrm{A}_{l}, \mathrm{C}_{l}$;

- $\Phi=\mathrm{B}_{l}, \mathrm{D}_{l}, s \leq l-2$, and the condition $\Lambda S_{l-s-1}$ is satisfied.

Then we have the decomposition

$$
G(\Phi, R)=P_{r} U_{r s}^{-} P_{s} .
$$

The present paper is the Diploma paper of the second author, under the supervision of the first author. In its sequel we prove similar results for some pairs of maximal parabolic subgroups in exceptional Chevalley groups. Due to the more complicated structure of unipotent radicals, new stability conditions may occur in these cases. Unfortunately, for exceptional groups we could not obtain similar decompositions for all pairs of maximal parabolic subgroups.

The paper is organized as follows. In $\S 1$ we give a general survey of the existing results on triangular and parabolic factorizations. In $\S \S 2-4$ we recall the basic notation pertaining to the split classical groups, parabolic subgroups, and stability conditions, respectively. The rest of the paper is devoted to the proof of our main results. Namely, in $\S 5$ we discuss the interrelation of stability conditions with the action of unipotent radicals, whereas in $\S 6$ the proof of Theorems 1 and 2 is reduced to the proof of the main lemma. This lemma is verified case by case in $\S \S 7-9$. Finally, in $\S 10$ we state some further problems related to parabolic factorizations, and their applications. 


\section{$\S 1$. Parabolic factorizations}

In the present section we give a brief survey of the triangular and parabolic factorizations that are generalized in the present paper.

- There is no doubt that the most important single fact pertaining to Chevalley groups over fields is the following Bruhat decomposition:

$$
G(\Phi, K)=B(\Phi, K) N(\Phi, K) U(\Phi, K)
$$

Recall that, here, $B(\Phi, R)$ is the standard Borel subgroup in $G(\Phi, R)$ containing the split maximal torus $T(\Phi, R)$,

$$
B(\Phi, R)=T(\Phi, R)\left\langle X_{\alpha}, \alpha \in \Phi^{+}\right\rangle,
$$

$U(\Phi, R)$ denotes the unipotent radical of $B(\Phi, R)$, and $N(\Phi, R)$ is the algebraic normalizer of $T(\Phi, R)$. When $R=K$ is a field with $|K| \geq 4$, the group $N(\Phi, K)$ coincides with the abstract normalizer of $T(\Phi, K)$ in $G(\Phi, K)$, while $N(\Phi, K) / T(\Phi, K) \cong W(\Phi)$. However, in general, the abstract normalizer of the torus can be considerably larger than $N(\Phi, R)$.

In view of the outstanding role played by the Bruhat decomposition in the structure theory and representation theory of algebraic groups over fields, it is natural to make an attempt to obtain similar results for more general commutative rings $R$. The Bruhat decomposition itself cannot be generalized to groups over rings, but in the simplest situations there are similar triangular factorizations, which are only marginally more complicated than the Bruhat decomposition.

- It is classically known that the groups over a semilocal ring $R$ admit the Gauss decomposition

$$
G(\Phi, R)=B(\Phi, R) U^{-}(\Phi, R) U(\Phi, R) .
$$

Here, $U^{-}(\Phi, R)$ denotes the unipotent radical of the standard Borel subgroup $B^{-}(\Phi, R)$ in $G(\Phi, R)$ opposite to $B(\Phi, K)$,

$$
B^{-}(\Phi, R)=T(\Phi, R)\left\langle X_{\alpha}, \alpha \in \Phi^{-}\right\rangle .
$$

Essentially, the Gauss decomposition follows already from the results of SGAIII; direct elementary proofs can be found, for instance, in the papers by Michael Stein [49], Eiichi Abe and Kazuo Suzuki [17. These results are based on elementary calculations, whereas the first named author and Eugene Plotkin [5] proposed yet another proof (of a more general fact), based on representation theory. In fact, as was noticed in [4, the Gauss decomposition can only be valid for rings of stable rank 1 , so that for the group $\operatorname{SL}(n, R)$ it holds if and only if $\operatorname{sr}(R)=1$.

- For stable classical groups, the Bruhat decomposition and Gauss decomposition admit a remarkable common generalization, namely, the Sharpe decomposition. Namely, the classical systems $X_{l}=\mathrm{A}_{l}, \mathrm{~B}_{l}, \mathrm{C}_{l}, \mathrm{D}_{l}$ of the same type admit embeddings $X_{l} \subseteq$ $X_{l+1}$. These embeddings beget regular embeddings of Chevalley groups $G\left(X_{l}, R\right) \longrightarrow$ $G\left(X_{l+1}, R\right)$. Passing to limits on the rank, we get the corresponding stable groups

$$
G(X, R)=\underset{\lim }{\longrightarrow}\left(X_{l}, R\right) .
$$

For instance, $G(A, R)=\mathrm{SL}(R)$ is the usual finitary special linear group, as considered in algebraic $K$-theory [1]. These limits agree with many habitual systems of subgroups. For example, it is clear what should be meant by $B(\mathrm{~B}, R)$ or $U(\mathrm{C}, R)$. In [43, 44] Sharpe proved the decomposition

$$
G(X, R)=B(X, R) N(X, R) U(X, R) U^{-}(X, R) .
$$


Obviously, this implies the decomposition

$$
G(X, R)=B(X, R) U^{-}(X, R) U(X, R) U^{-}(X, R) .
$$

On the other hand, in general, for rings of dimension at least 1 , the Chevalley groups of finite rank $G(\Phi, R)$ themselves do not admit any similar decomposition in terms of elementary generators. This is due to the following circumstances.

- First, the group $G(\Phi, R)$ itself may fail to be generated by elementary generators. Thus, instead of $G(\Phi, R)$, one should either consider its elementary subgroup $E(\Phi, R)$, or else, allow semisimple generators together with the unipotent ones, and consider the subgroup

$$
G_{0}(\Phi, R)=E(\Phi, R) T(\Phi, R) .
$$

- However, even in the case where the group $G(\Phi, R)$ is generated by the elementary generators, it may fail to have bounded width with respect to these generators. Wilberd van der Kallen [34] observed that this is the case already for the Euclidean ring $R=\mathbb{C}[x]$. Quite amazingly, already the special linear group $\mathrm{SL}(3, \mathbb{C}[x])$ does not have bounded width with respect to the elementary transvections. See also [27], where a slightly more explicit proof of a slightly more general fact can be found.

This means that nontrivial generalizations of the Bruhat and Gauss decompositions can only be valid under some additional assumptions on the ring and/or should be stated in different terms.

Among such generalizations, the parabolic factorizations (in other words, the decompositions stated in terms of parabolic subgroups and stability conditions) are most widely known. Clearly, before [38, 50, no "parabolic subgroups" were explicitly mentioned in this context, and all decompositions were described as explicit matrix factorizations.

We mention two most eminent decompositions of this kind. For the groups $\operatorname{GL}(n, R)$, $\mathrm{SL}(n, R)$, and $E(n, R)$, they are stated in terms of the stable rank $\operatorname{sr}(R)$ of the ring $R$. Roughly speaking, these are the weakest forms of Bruhat and Gauss decompositions, which survive for arbitrary finite-dimensional rings.

- The proof of surjective stability for the functor $K_{1}$ relies on the remarkable BassKolster decomposition that asserts that for $n>\operatorname{sr}(R)$ we have

$$
\mathrm{GL}(n, R)=\mathrm{GL}(n-1, R) U_{n-1} U_{n-1}^{-} U_{n-1} U_{n-1}^{-} .
$$

As above, the group $\operatorname{GL}(n-1, R)$ is viewed as a $\operatorname{subgroup~of~} \mathrm{GL}(n, R)$ via the stability map

$$
\mathrm{GL}(n-1, R) \longrightarrow \mathrm{GL}(n, R), \quad g \mapsto\left(\begin{array}{ll}
g & 0 \\
0 & 1
\end{array}\right),
$$

whereas the groups $U_{n-1}$ and $U_{n-1}^{-}$are the unipotent radicals of the opposite parabolic subgroups $P_{n-1}$ and $P_{n-1}^{-}$, respectively. The definitions of these and all other occurring subgroups are recalled in $\S 2$. This decomposition was discovered by Hyman Bass [22] and systematically studied by Manfred Kolster [36.

- On the other hand, the proof of injective stability for the functor $K_{1}$ and that of surjective stability for the functor $K_{2}$ relies on the Dennis-Vaserstein decomposition, asserting that for $n>\operatorname{sr}(R)+1$ one has

$$
E(n, R)=P X_{n 1} Q,
$$

where $P=\mathrm{EP}_{1}$ and $Q=\mathrm{EP}_{n-1}$ are the elementary parabolic subgroups, and $X_{n 1}$ is a unipotent root subgroup. For the group $E(n, R)$ itself, this decomposition was first noted by Keith Dennis [25, in the context of surjective stability for $K_{2}$. Soon thereafter, Leonid Vaserstein [10] used similar decompositions for other classical groups, in the context of injective stability for $K_{1}$. 
Properly speaking, Dennis and Vaserstein used a slightly weaker decomposition, with some additional factors. In the above precise form, for the first time it was stated by Andrei Suslin and Marat Tulenbaev in the remarkable paper [16] devoted to injective stability for the $K_{2}$-functor.

Subsequently Michael Stein [50] and Eugene Plotkin [13, 14, 40, 41] generalized decomposition of this type to other Chevalley groups. This line of development was summarized in the paper [42], where particularly easy proofs of these generalizations were presented; unfortunately, this paper is still unpublished.

In general, these generalizations invoked some stronger stability conditions, rather than the stable rank itself. One such prominent condition is the absolute stable rank $\operatorname{asr}(R)$, see [28, 50, 37, 47]. In particular, results in terms of the absolute stable rank superceeded the classical results by Anthony Bak [18] and Hyman Bass [23], stated in terms of dimension. Stein's paper [50] emphasizes another significant advantage of the use of $\operatorname{asr}(R)$, which allows us to treat all cases uniformly.

However, in general, for a very long time, no natural stability conditions have been detected, which would play the same role for other groups as the usual stable rank does for $\operatorname{SL}(n, R)$. The quest for such natural conditions went on for several decades, with some fairly successful attempts. Thus, Plotkin [14, 40] used analogs of Vaserstein conditions $V_{l}$, see [8]. Gerhard Habdank [29, 30] used unitary stable rank. Their results covered the classical results stated in terms of dimension, but not each other, with no definitive results in sight.

In the papers [19, 20, inspired by [42, Anthony Bak, Viktor Petrov, and Tang Guoping obtained generalizations of the Dennis-Vaserstein decomposition to unitary groups. The main contribution of those papers is in the introduction of new stability conditions defined in terms of the action of unipotent radicals of parabolic subgroups, most notably, the $\Lambda$ stable rank. Today, it became apparent that these conditions, rather than any previously known ones, are the genuine higher analogs of the usual stable rank.

However, since all these papers were expressly focused upon the proof of stability theorems, they only considered pairs of terminal maximal parabolic subgroups, corresponding to the end nodes of the Dynkin diagram. The reason is that this choice entails the weakest possible stability conditions on the ring $R$.

Following [6], in the present paper we initiate a systematic study of Bass-Kolster type factorizations, and of Dennis-Vaserstein type factorizations, for all pairs of maximal parabolic subgroups, not necessarily terminal ones. In particular, a whole range of different stability conditions crop up. Due to the more complicated structure of the unipotent radicals, most of these stability conditions are new. However, for classical groups the unipotent radicals of maximal parabolic subgroups have class at most 2 . Therefore, in this setting it suffices to deal with the $\Lambda$-stable rank introduced by Bak, Petrov, and Tang.

\section{$\S 2$. Split Classical Groups}

In the present section we very briefly recall the principal notation used in the rest of the paper. Mostly, it is utterly standard and closely follows that in the preceding publications. One can find most of the necessary background on the theory of classical groups over rings in the marvelous book [31] by Alex Hahn and Timothy O'Meara.

Let $R$ be an associative ring with 1 , and let $R^{*}$ be its multiplicative group. In the main results of the present paper we assume that the ring $R$ is commutative. As usual, $M(m, n, R)$ is the additive group of $(m \times n)$-matrices, and $M(n, R)=M(n, n, R)$ is the ring of square matrices of degree $n$. Next, $\operatorname{GL}(n, R)=M(n, R)^{*}$ and $\operatorname{SL}(n, R)$ denote the general linear group and the special linear group of degree $n$ over $R$, respectively. 
By $a_{i j}$ we denote the entry of a matrix $a$ at the position $(i, j)$; thus, $a=\left(a_{i j}\right)$. $a^{-1}=\left(a_{i j}^{\prime}\right)$ denotes the inverse of $a$, and $a^{t}$ denotes its transpose. Also, $a_{* j}$ is the $j$ th column of a matrix $a$, and $a_{i *}$ is its $i$ th row. As usual, $e$ is the identity matrix, while $e_{i j}$ is the standard matrix unit, i.e., the matrix that has 1 at the position $(i, j)$ and zeroes elsewhere.

As usual, $t_{i j}(\xi)=e+\xi e_{i, j}$, where $1 \leq i \neq j \leq n, \xi \in R$, denotes an elementary transvection, and $X_{i j}=\left\{t_{i j}(\xi), \xi \in R\right\}$ denotes a root subgroup.

Consider the free right $R$-module $R^{n}$ of rank $n$. The elements of $R^{n}$ are construed as columns of height $n$ with components in $R$. Let $e_{1}, \ldots, e_{n}$ be the standard base of $R^{n}$. Similarly, the free left $R$-module ${ }^{n} R$ of rank $n$ is interpreted as the module of rows of length $n$ with components in $R$. Let $f_{1}, \ldots, f_{n}$ be its standard base.

We pass to a description of other split classical groups. Denote by $l=\lfloor n / 2\rfloor$ the integral part of $n / 2$. As everybody knows, one should number the columns and rows of matrices in these groups as follows:

$$
\begin{array}{ll}
1, \ldots, l,-l, \ldots,-1 & \text { if } n=2 l \text { is even, } \\
1, \ldots, l, 0,-l, \ldots,-1 & \text { if } n=2 l+1 \text { is odd. }
\end{array}
$$

All other occurring objects are indexed in the same handy way. Thus, now the base of $R^{n}$ will be written as

$$
\begin{array}{ll}
e_{1}, \ldots e_{l}, e_{-l}, \ldots, e_{-1} & \text { if } n=2 l \text { is even, } \\
e_{1}, \ldots e_{l}, e_{0}, e_{-l}, \ldots, e_{-1} & \text { if } n=2 l+1 \text { is odd }
\end{array}
$$

Denote by $\varepsilon_{i}$ the sign of $i$, so that $\varepsilon_{i}=1$ for $i=1, \ldots, l$ and $\varepsilon_{i}=-1$ for $i=-1, \ldots,-l$.

Let $\operatorname{sdiag}\left(\lambda_{1}, \ldots, \lambda_{n}\right)$ stand for the matrix in $\operatorname{GL}(n, R)$ with the skew diagonal entries $\lambda_{1}, \ldots, \lambda_{n}$, in the North-East to South-West direction, and with zeros at all other positions. Set

$$
F=F_{2 l}=\operatorname{sdiag}(1, \ldots, 1,-1, \ldots,-1),
$$

where both the total number of 1's and the total number of -1 's are equal to $l$. In other words, the entries of $F=\left(F_{i j}\right)$ are defined by the condition $F_{i j}=\varepsilon_{i} \delta_{i,-j}$. Next, we set

$$
f=f_{2 l}=\operatorname{sdiag}(0, \ldots, 0,1, \ldots, 1),
$$

where both the total number of 0 's and the total number of 1 's are equal to $l$ when $n$ is even, and

$$
f=f_{2 l+1}=\operatorname{sdiag}(0, \ldots, 0,1,1, \ldots, 1),
$$

where the total number of 0 's is equal to $l$, whereas the total number of 1 's is equal to $l+1$, when $n$ is odd.

Consider the symplectic inner product $B$ on $R^{2 l}$ with the Gram matrix $F_{2 l}$. By definition, the symplectic group $\operatorname{Sp}(2 l, R)$ consists of all matrices $g \in \operatorname{GL}(2 l, R)$ that preserve the inner product $B$, in the usual sense that

$$
B(g u, g v)=B(u, v), \quad u, v \in R^{2 l} .
$$

In other words, a matrix $g \in \mathrm{SL}(2 l, R)$ belongs to the group $\operatorname{Sp}(2 l, R)$ if and only if $g^{t} F g=F$ or, what is the same, if

$$
g_{i j}^{\prime}=\varepsilon_{i} \varepsilon_{j} g_{-j,-i} \quad \text { for all } i, j=1, \ldots,-1 .
$$

From the viewpoint of algebraic groups, the group $\operatorname{Sp}(2 l, R)$ is the simply connected Chevalley group of type $\mathrm{C}_{l}$.

As usual, an elementary symplectic transvection is one of the matrices $T_{i j}(\xi), \xi \in R$, $i \neq j$, of the form

$$
T_{i j}(\xi)=T_{-j,-i}(-\xi)=e+\xi e_{i j}-\varepsilon_{i} \varepsilon_{j} \xi e_{-j,-i}, \quad i \neq \pm j,
$$


or of the form

$$
T_{i,-i}(\xi)=e+\xi e_{i,-i} .
$$

From the viewpoint of Chevalley groups, these elements are unipotent short root elements and long root elements, respectively, and the group they generate is called the elementary Chevalley group $E\left(\mathrm{C}_{l}, R\right)$. In the classical terminology, this last group is known as the elementary symplectic group $\operatorname{Ep}(2 l, R)$.

In the above notation, the root unipotents corresponding to the fundamental roots are described as follows:

$$
x_{\alpha_{i}}(\xi)=T_{i, i+1}(\xi), \quad \text { for } i=1, \ldots, l-1, \quad x_{\alpha_{l}}(\xi)=T_{l,-l}(\xi) .
$$

The root unipotents corresponding to the negative fundamental roots are obtained by transposition.

Now, let $q$ be the quadratic form on $R^{n}$ defined by $v \mapsto v^{t} f_{n} v$. By definition, the orthogonal group $\mathrm{SO}(n, R)$ consists of the matrices $g \in \mathrm{GL}(n, R)$ that preserve the quadratic form $q$. Define a symmetric form by

$$
h(u, v)=q(u+v)-q(u)-q(v) .
$$

A fortiori, the elements of $\mathrm{SO}(n, R)$ preserve this form. The Gram matrix $H_{n}$ of the form $h$ is $H_{n}=f_{n}+f_{n}{ }^{t}$, so that $g^{t} h_{n} g=h_{n}$. This implies polynomial equations on matrix entries of a matrix $g \in \mathrm{SO}(n, R)$, namely,

$$
g_{i j}^{\prime}=g_{-j,-i} \quad \text { for } \quad i, j=1, \ldots, l,-l, \ldots,-1 .
$$

In the case where $n=2 l$, these equations completely describe the group $\mathrm{SO}(2 l, R)$. From the viewpoint of algebraic groups, the group $\mathrm{SO}(n, R)$ is a Chevalley group of type $\mathrm{B}_{l}$ or $\mathrm{D}_{l}$, respectively, depending on whether $n=2 l+1$ or $n=2 l$.

As in the symplectic case, an elementary orthogonal transvection is one of the matrices $T_{i j}(\xi), \xi \in R, i \neq \pm j$, of the form

$$
T_{i j}(\xi)=T_{-j,-i}(-\xi)=e+\xi e_{i j}-\xi e_{-j,-i}, \quad i \neq \pm j
$$

or of the form

$$
T_{0,-i}(\xi)=e+\xi e_{0,-i}-2 \xi e_{i, 0}-\xi^{2} e_{i,-i}, \quad i=1, \ldots, l .
$$

From the viewpoint of Chevalley groups, these elements are unipotent long root elements and short root elements, respectively, and the group they generate is called the elementary Chevalley group $E(\Phi, R)$. Obviously, short root elements only occur in the case of $\Phi=\mathrm{B}_{l}$.

In the above notation, the root unipotents corresponding to the fundamental roots, except for the last one, are described as follows:

$$
x_{\alpha_{i}}(\xi)=T_{i, i+1}(\xi), \quad \text { for } i=1, \ldots, l-1 .
$$

In its turn, the root element corresponding to the last fundamental root equals

$$
\begin{array}{ll}
x_{\alpha_{l}}(\xi)=T_{0,-l}(\xi) & \text { for } \Phi=\mathrm{B}_{l}, \\
x_{\alpha_{l}}(\xi)=T_{l-1,-l}(\xi) & \text { for } \Phi=\mathrm{D}_{l} .
\end{array}
$$

Again, the root unipotents corresponding to the negative fundamental roots are obtained by transposition. From the classical viewpoint, these elements generate the elementary orthogonal group $\operatorname{EO}(n, R)$. Over a field, this last group is also known as the kernel of the spin norm $\Omega(n, R)$.

In the sequel, we use these explicit identifications of the classical groups, and of their unipotent root elements, without any further notice. 


\section{$\S 3$. Standard PARABOlic SUbGroups}

The main results of the present paper are stated in terms of the maximal standard parabolic subgroups.

Let $i=1, \ldots, l$. Denote by

$$
S_{i}=\left\{\alpha \in \Phi, \alpha=\sum_{k} m_{k}(\alpha) \alpha_{k}, m_{i}(\alpha) \geq 0\right\}
$$

the $i$ th maximal standard parabolic subset of $\Phi$. As usual, its reductive part $\Delta_{i}$ and its special part $\Sigma_{i}$ are defined as

$$
\begin{aligned}
& \Delta_{i}=\left\{\alpha \in \Phi, \alpha=\sum_{k} m_{k}(\alpha) \alpha_{k}, m_{i}(\alpha)=0\right\}, \\
& \Sigma_{i}=\left\{\alpha \in \Phi, \alpha=\sum_{k} m_{k}(\alpha) \alpha_{k}, m_{i}(\alpha)>0\right\} .
\end{aligned}
$$

The opposite parabolic subset and its special part are defined similarly:

$$
\begin{aligned}
& S_{i}^{-}=\left\{\alpha \in \Phi, \alpha=\sum_{k} m_{k}(\alpha) \alpha_{k}, m_{i}(\alpha) \leq 0\right\}, \\
& \Sigma_{i}^{-}=\left\{\alpha \in \Phi, \alpha=\sum_{k} m_{k}(\alpha) \alpha_{k}, m_{i}(\alpha)<0\right\} .
\end{aligned}
$$

We denote by $\mathrm{EP}_{i}$ the $i$ th elementary maximal standard parabolic subgroup of the elementary group $E(\Phi, R)$. By definition,

$$
\mathrm{EP}_{i}=\left\langle x_{\alpha}(\xi), \alpha \in S_{i}, \xi \in R\right\rangle .
$$

Levi's decomposition asserts that the group $\mathrm{EP}_{i}$ can be expressed as the semidirect product $\mathrm{EP}_{i}=\mathrm{EL}_{i} \wedge U_{i}$ of the elementary Levi subgroup $\mathrm{EL}_{i}$ and the unipotent radical $U_{i}$, where

$$
\mathrm{EL}_{i}=\left\langle x_{\alpha}(\xi), \alpha \in \Delta_{i}, \xi \in R\right\rangle, \quad U_{i}=\left\langle x_{\alpha}(\xi), \alpha \in \Sigma_{i}, \xi \in R\right\rangle .
$$

With a parabolic set of roots $S_{i}$ we can naturally associate also an algebraic subgroup $P_{i}$, which is called the maximal standard parabolic subgroup of $G(\Phi, K)$; see 24,38 . By the very definition, $P_{i}$ contains the Levi subgroup $\mathrm{EP}_{i}$, and is in fact its Zariski closure. The subgroup $P_{i}$ also admits the Levi decomposition $P_{i}=L_{i} \curlywedge U_{i}$, where $L_{i}=G\left(\Delta_{i}, R\right)$ is a reductive algebraic group of type $\Delta_{i}$. The elementary subgroup of $L_{i}$ coincides with $\mathrm{EL}_{i}=E(\Delta, R)$.

Together with the subgroups $P_{i}$ and $\mathrm{EP}_{i}$, we shall consider also the opposite subgroups $P_{i}^{-}$and $\mathrm{EP}_{i}^{-}$, and their unipotent radical $U_{i}^{-}$. These subgroups are defined similarly, only the root sets $S_{i}$ and $\Sigma_{i}$ are replaced by their opposite root sets. Set

$$
U_{i j}=U_{i} \cap U_{j}, \quad U_{i j}^{-}=U_{i}^{-} \cap U_{j}^{-} .
$$

We describe the matrix realizations of parabolic subgroups. First, consider the case where $\Phi=A_{l}$. We have $E(\Phi, R)=E(n, R)$ for $n=l+1$. In this case $x_{\alpha_{r}}(\xi)=t_{r, r+1}(\xi)$. In the sequel, it will be handy to write elements of the group $E(n, R)$ as block matrices, in accordance with the partition $(s, r-s, n-r)$ of degree $n$. In particular, arbitrary elements $p \in P_{r}$ and $q \in P_{s}$ have the form

$$
p=\left(\begin{array}{ccc}
p^{11} & p^{12} & p^{1,} \\
p^{21} & p^{22} & p^{23} \\
0 & 0 & p^{33}
\end{array}\right), \quad q=\left(\begin{array}{ccc}
q^{11} & q^{12} & q^{13} \\
0 & q^{22} & q^{23} \\
0 & q^{32} & q^{33}
\end{array}\right)
$$

The elements of the opposite parabolic subgroups $p \in P_{r}^{-}$and $q \in P_{s}^{-}$have the form

$$
p=\left(\begin{array}{ccc}
p^{11} & p^{12} & 0 \\
p^{21} & p^{22} & 0 \\
p^{31} & p^{32} & p^{33}
\end{array}\right), \quad q=\left(\begin{array}{ccc}
q^{11} & 0 & 0 \\
q^{21} & q^{22} & q^{23} \\
q^{31} & q^{32} & q^{33}
\end{array}\right)
$$


whereas the elements of the Levi subgroups $p \in L_{r}, q \in L_{s}$ have the form

$$
p=\left(\begin{array}{ccc}
p^{11} & p^{12} & 0 \\
p^{21} & p^{22} & 0 \\
0 & 0 & p^{33}
\end{array}\right), \quad q=\left(\begin{array}{ccc}
q^{11} & 0 & 0 \\
0 & q^{22} & q^{23} \\
0 & q^{32} & q^{33}
\end{array}\right) .
$$

On the other hand, for the same partition the unipotent radicals are of the form

$$
U_{r}=\left(\begin{array}{ccc}
e & 0 & * \\
0 & e & * \\
0 & 0 & e
\end{array}\right), \quad U_{s}=\left(\begin{array}{ccc}
e & * & * \\
0 & e & 0 \\
0 & 0 & e
\end{array}\right), \quad U_{r s}=\left(\begin{array}{ccc}
e & 0 & * \\
0 & e & 0 \\
0 & 0 & e
\end{array}\right),
$$

whereas the opposite unipotents radicals are of the form

$$
U_{r}^{-}=\left(\begin{array}{ccc}
e & 0 & 0 \\
0 & e & 0 \\
* & * & e
\end{array}\right), \quad U_{s}^{-}=\left(\begin{array}{ccc}
e & 0 & 0 \\
* & e & 0 \\
* & 0 & e
\end{array}\right), \quad U_{r s}^{-}=\left(\begin{array}{ccc}
e & 0 & 0 \\
0 & e & 0 \\
* & 0 & e
\end{array}\right),
$$

respectively.

An explicit matrix form of the relevant maximal parabolic subgroups in symplectic and orthogonal groups will be reproduced in $\S \S 8,9$.

\section{$\S 4$. Stability CONDITIONS}

Here, we describe the stability conditions used in the present paper. The simplest and most commonly known among these conditions is the usual stable rank, introduced by Hyman Bass in 1964; see [22].

A row $\left(a_{1}, \ldots, a_{n}\right) \in{ }^{n} R$ is said to be unimodular if its components $a_{1}, \ldots, a_{n}$ generate $R$ as a right ideal,

$$
a_{1} R+\cdots+a_{n} R=R
$$

or, what is the same, if there exist $b_{1}, \ldots, b_{n} \in R$ such that

$$
a_{1} b_{1}+\cdots+a_{n} b_{n}=1 \text {. }
$$

The stable rank of the ring $R$ is the smallest $n$ such that every unimodular row $\left(a_{1}, \ldots, a_{n+1}\right)$ of length $n+1$ is stable. In other words, there exist elements $b_{1}, \ldots, b_{n} \in R$ such that the row

$$
\left(a_{1}+a_{n+1} b_{1}, a_{2}+a_{n+1} b_{2}, \ldots, a_{n}+a_{n+1} b_{n}\right)
$$

of length $n$ is unimodular. If no such $n$ exists, we write $\operatorname{sr}(R)=\infty$. Bass denoted stability of unimodular rows of length $n+1$ by $\mathrm{SR}_{n+1}(R)$. It is easily seen that condition $\mathrm{SR}_{m}(R)$ implies condition $\mathrm{SR}_{n}(R)$ for all $n \geq m$, so that the stable rank is well defined: if $n>\operatorname{sr}(R)$, then every unimodular row of length $n$ is stable.

Clearly, when $n>\operatorname{sr}(R)+1$, the process of shortening a unimodular row can be iterated. Thus, the following lemma is obvious; its proof can be found, e.g., in [9].

Lemma 1. Let $n>m \geq \operatorname{sr}(R)$, and let $\left(a_{1}, \ldots, a_{n}\right) \in{ }^{n} R$ be a unimodular row of length $n$. Then there exists a matrix $b=\left(b_{i j}\right) \in M(n-m, m, R)$ such that the row

$$
\left(a_{1}, \ldots, a_{m}\right)+\left(a_{m+1}, \ldots, a_{n}\right) b
$$

is unimodular.

The most familiar variation of stable rank that works for other classical groups is the absolute stable rank. For commutative rings, this condition was introduced by David Estes and Jack Ohm [28], whereas Michael Stein [50] discovered its relevance in the study of orthogonal groups and exceptional groups. See also [37, 45, 46, 47, 13, 15, 12] for estimates of the absolute stable rank, and its noncommutative generalizations. 
For a row $\left(a_{1}, \ldots, a_{n}\right) \in{ }^{n} R$, we denote by $J\left(a_{1}, \ldots, a_{n}\right)$ the intersection of the maximal ideals of $R$ containing $a_{1}, \ldots, a_{n}$. In particular, a row is unimodular if and only if $J\left(a_{1}, \ldots, a_{n}\right)=R$.

A commutative ring $R$ is said to satisfy condition $\operatorname{ASR}_{n+1}$ if for any row $\left(a_{1}, \ldots, a_{n+1}\right)$ of length $n+1$ there exist elements $b_{1}, \ldots b_{n} \in R$ such that

$$
J\left(a_{1}+a_{n+1} b_{1}, \ldots, a_{n}+a_{n+1} b_{n}\right)=J\left(a_{1}, \ldots, a_{n+1}\right) .
$$

It is easy to show that condition $\operatorname{ASR}_{m}(R)$ implies condition $\operatorname{ASR}_{n}(R)$ for all $n \geq m$. The absolute stable rank $\operatorname{asr}(R)$ of the ring $R$ is the smallest natural $n$ for which condition $\operatorname{ASR}_{n+1}(R)$ is fulfilled. Clearly, $\operatorname{sr}(R) \leq \operatorname{asr}(R)$.

Next, following Bak, Petrov, and Tang, we define the stable rank of a form ring. Namely, let $R$ be an arbitrary associative ring with 1, and let $h: a \mapsto \bar{a}$ be an antiinvolution with symmetry $\lambda$. In other words, $\overline{\bar{a}}=\lambda a \bar{\lambda}$. Let $\Lambda$ be some $\lambda$-form parameter of the ring $R$. The ring $R$ together with an anti-involution and a form parameter is called a form ring; see [11, 12, 18, 22, 23, 31, 32, 35].

In the present section, it is convenient to assume that the columns of a matrix in $M(n, R)$ are numbered as $-n, \ldots,-1$, while the rows are numbered in the usual way. Define an involution on $M(n, R)$ as follows: for $a \in M(n, R)$, set $a \mapsto \bar{a}=a_{i,-j}^{\prime}$, where $a_{i,-j}^{\prime}=\overline{a_{j,-i}}$. Let $M_{n}(\bar{\Lambda})$ denote the set of matrices $b \in M(n, R)$ such that $b=-\bar{\lambda} \cdot \bar{b}$ and, moreover, $b_{i,-i} \in \bar{\Lambda}$ for all $i=1 \ldots n$.

By definition, the ring $R$ satisfies the stability condition $\Lambda S_{m}$ if sr $R \leq m$ and, moreover, for any unimodular row

$$
a=\left(a^{+}, a^{-}\right)=\left(a_{1}, \ldots, a_{m+1}, a_{-(m+1)}, \ldots, a_{-1}\right)
$$

of length $2(m+1)$ there exists a matrix $b \in M_{m+1}(\bar{\Lambda})$ such that $a^{+} b+a^{-}$is a unimodular row of length $m+1$.

Lemma 2. Condition $\Lambda S_{m}$ implies $\Lambda S_{n}$ for $n>m$.

In particular, this lemma allows us to define the stable rank $\operatorname{sr}(R, \Lambda)$ of a form $\operatorname{ring} R$ as the smallest $n$ such that $\Lambda S_{n}$ is satisfied.

The above definition was first introduced by Bak and Tang in their paper [20], in the context of Hermitian groups, and was subsequently used by Petrov in his study of generalized unitary groups (which simultaneously cover the usual unitary and Hermitian groups) in his Thesis [12].

Now, we recall the interrelations of $\Lambda S_{m}$ with other stability conditions studied previously.

A form ring $R$ is said to satisfy condition $\Lambda \mathrm{US}_{m}$ if $\mathrm{sr} R \leq m$ and for any unimodular column $u \in R^{2 m}$ there exists a column $v \in R^{2 m}$ such that $v^{t} H_{2 m} u=1$ and $v^{t} f_{2 m} v \in \Lambda$.

The proof of the following result can be found in [12, 20].

Lemma 3. Condition $\Lambda S_{m}$ is satisfied in any of the following cases:

- condition $\mathrm{ASR}_{m}$ is fulfilled,

- condition $\Lambda \mathrm{US}_{m}$ is fulfilled.

In the special case where the ring $R$ is commutative, the involution $h$ equals $x \mapsto-x$, and $\Lambda=0$, we have

$$
\operatorname{sr}(R, R)=\operatorname{usr}(R, R)=\operatorname{sr}(R) .
$$

Thus, for the symplectic group the usual stable rank condition suffices in the statements of all results.

Now we turn to the opposite case, where the ring is $R$ commutative, the involution $h$ equals $x \mapsto-x$, and $\Lambda=0$. If we view $\left(R^{n}, q\right)$ as a quadratic space, the unitary group of 
this space in the sense of 20] coincides with the orthogonal group $\mathrm{SO}(n, R)$ as defined above.

For the future use (rather than for the use in the present paper), we observe that the multiple stable rank condition introduced by Wilberd van der Kallen [33] is also described quite naturally in terms of the action of the unipotent radical of the maximal parabolic subgroup $P_{m}$ in $\operatorname{SL}(n, R)$. In these terms, the usual stable rank corresponds to the special case where $P_{m}=P_{1}$.

\section{$\S 5$. The ACTION OF UNIPOTENT RADiCALS}

Here, we specify the interrelation of the stability conditions introduced in the preceding section with the action of unipotent radicals of parabolic subgroups.

Lemma 4. Suppose the ring $R$ satisfies condition $\Lambda S_{m-1}$, where $\Lambda=0$ and the involution is defined by $\bar{x}=-x$. Then for every unimodular row

$$
\left(u_{1}, \ldots, u_{m}, u_{0}, u_{-m}, \ldots, u_{-1}\right)
$$

we can find a matrix $g \in E\left(B_{m}, R\right)$ of the form

$$
\left(\begin{array}{lll}
e & * & * \\
0 & 1 & * \\
0 & 0 & e
\end{array}\right)
$$

such that the row $\left(v_{-m}, \ldots v_{-1}\right)$ consisting of the components of the vector $v=g u$ is unimodular.

Proof. Since sr $R \leq m$ by assumption, there exist $a_{-m}, \ldots a_{-1}$ such that the row

$$
\left(u_{1}, \ldots, u_{m}, u_{-m}+a_{-m} u_{0}, \ldots, u_{-1}+a_{-1} u_{0}\right)
$$

is unimodular. Consider

$$
g_{1}=\prod_{i=-m}^{-1} T_{0, i}\left(a_{i}\right), \quad u^{\prime}=g_{1} u
$$

then the row $\left(u_{1}^{\prime}, \ldots, u_{m}^{\prime}, u_{-m}^{\prime}, \ldots, u_{-1}^{\prime}\right)$ is also unimodular. It remains to take a skew persymmetric matrix $b \in M_{m}(R)$ such that

$$
\left(u_{1}^{\prime}, \ldots, u_{m}^{\prime}\right) b+\left(u_{-m}^{\prime}, \ldots, u_{-1}^{\prime}\right)
$$

is unimodular, and set

$$
g_{2}=\left(\begin{array}{lll}
e & 0 & b \\
0 & 1 & 0 \\
0 & 0 & e
\end{array}\right)=\prod_{1 \leq i<j \leq m} T_{i,-j}\left(b_{i,-j}\right), v=g_{2} u^{\prime} .
$$

Clearly, $g=g_{1} g_{2}$ is the desired matrix.

Lemma 5. Suppose the ring $R$ satisfies the condition $\mathrm{sr} R \leq m$. Then for every unimodular row $\left(u_{1}, \ldots, u_{m}, u_{-m}, \ldots, u_{-1}\right)$ we can find a matrix $g \in E\left(\mathrm{C}_{m}, R\right)$ of the form $g=$ $\left(\begin{array}{ll}e & * \\ 0 & e\end{array}\right)$ such that the row $\left(v_{-m}, \ldots, v_{-1}\right)$ consisting of the components of the vector $v=g u$ is unimodular.

Proof. We view the commutative ring $R$ as a form ring with the trivial involution and the form parameter $\Lambda=R$. Clearly, when $\Lambda=R, \Lambda U S_{m}$ follows from the condition sr $R \leq m$. Thus, by Lemma 2 , the ring $R$ satisfies condition $\Lambda S_{m}$. Then there is a 
persymmetric matrix $b$ such that $\left(u_{1}, \ldots, u_{m}\right) b+\left(u_{-m}, \ldots, u_{-1}\right)$. As a required $g$ we can take

$$
g=\prod_{1 \leq i \leq j \leq m} T_{i,-j}\left(b_{i,-j}\right)
$$

\section{$\S 6$. THE BASIC REDUCTION}

In the present section we show that, modulo some common knowledge from the theory of algebraic groups, the proof of our main theorems can be reduced to the existence of some subsets in parabolic subgroups, with the following properties.

- On one hand, replacing parabolic subgroups by these subsets, we do not change the factorization.

- On the other hand, modulo this replacement, the verification that the above decomposition withstands multiplication by root subgroups becomes much easier technically.

The following obvious fact drastically simplifies all subsequent calculations. Its proof immediately follows from the Chevalley commutator formula.

Lemma 6. For each $1 \leq i \leq l$, the group $E(\Phi, R)$ is generated by the elementary parabolic subgroup $\mathrm{EP}_{i}$ and the root subgroup $X_{-\alpha_{i}}$.

Thus, to verify that a nonempty subset $Y \subseteq E(\Phi, R)$ coincides with the whole group $E(\Phi, R)$, it suffices to check that $\mathrm{EP}_{i} \cdot Y \subseteq Y$ and $X_{-\alpha_{i}} Y \subseteq Y$.

A similar result is valid for parabolic subgroups themselves, under some additional assumptions that guarantee surjective stability for $\mathrm{K}_{1}$. Observe that the proof of injective stability for $K_{1}$ merely relies on (some special cases of) Theorem 1, which depends on Lemma 6. This theorem invokes no stability conditions whatsoever. Theorem 2 is never used in this proof. The only place in the proof of Theorem 1 that invokes stability conditions is the construction of the set $\widetilde{P}$ in the following three sections.

Lemma 7. Suppose $1 \leq i \leq l$ and one of the following conditions is fulfilled:

- $\operatorname{sr}(R) \leq \max (i-1, l-i)$ for $\Phi=\mathrm{A}_{l}$;

- $\operatorname{sr}(R) \leq l-i$ for $\Phi=\mathrm{C}_{l}, i \leq l-1$;

- $\Lambda S_{l-s}$ for $\Phi=\mathrm{B}_{l}, \mathrm{D}_{l}, i \leq l-1$.

Then $G$ is generated by the subgroups $P_{i}$ and $X_{-\alpha_{i}}$.

Proof. Observe that the subgroup generated by $P_{i}$ and $X_{-\alpha_{i}}$ contains $E(\Phi, R)$, and therefore coincides with $G(\Phi, R)$, whenever the stability homomorphism

$$
\phi: \mathrm{K}_{1}\left(\Delta_{i}, R\right) \rightarrow \mathrm{K}_{1}\left(\Phi_{l}, R\right)
$$

is surjective. It remains to observe that the system $\Psi=\Phi_{l-i}$ (and if $\Phi=\mathrm{A}_{l}$, the system $\left.\Psi=\mathrm{A}_{i-1}\right)$ naturally embeds in $\Delta_{i}$. Now, Theorem 3 of [12] implies that the stability homomorphism $\mathrm{K}_{1}(\Psi, R) \rightarrow \mathrm{K}_{1}\left(\Phi_{l}, R\right)$ is surjective under the above assumption on the $\operatorname{ring} R$. A fortiori, the homomorphism $\phi$ is also surjective under the same assumption.

The following claims are the key steps in the proofs of Theorems 1 and 2, respectively. Observe that their proofs do not invoke anything whatsoever, apart from the simplest general facts, such as the Levi decomposition for parabolic subgroups! Namely, fix some $1 \leq i, j \leq l$ and consider the products on the right-hand sides of the factorizations we need to prove to establish Theorems 1 and 2 :

$$
\mathrm{EY}_{i j}=\mathrm{EP}_{i} \cdot U_{i j}^{-} \cdot \mathrm{EP}_{j}, \quad Y_{i j}=P_{i} U_{i j}^{-} P_{j}
$$


Lemma 8. Suppose that there exists a subset $\widetilde{P} \subseteq \mathrm{EP}_{i}$ with the following properties.

- For every $q \in \mathrm{EP}_{i}$ there exists an element $x \in \mathrm{EL}_{i} \cap \mathrm{EP}_{j}$ such that $q x \in \widetilde{P}$.

- The inclusion $X_{-\alpha_{i}} \widetilde{P} \subseteq \mathrm{EP}_{i} \cdot \mathrm{EP}_{j}^{-}$holds.

Then

$$
X_{-\alpha_{i}} \cdot \mathrm{EY} \subseteq \mathrm{EY} .
$$

Lemma 9. Suppose that there exists a subset $\widetilde{P} \subseteq P_{i}$ with the following properties.

- For every $q \in P_{i}$ there exists an element $x \in \mathrm{EL}_{i} \cap \mathrm{EP}_{j}$ such that $q x \in \widetilde{P}$.

- The inclusion $X_{-\alpha_{i}} \widetilde{P} \subseteq P_{i} \cdot \mathrm{EP}_{j}^{-}$holds.

Then

$$
X_{-\alpha_{i}} Y \subseteq Y .
$$

The proofs of Lemmas 8 and 9 are quite similar. As a pattern, we present a proof of the slightly more difficult Lemma 9.

Proof. Express an arbitrary element $y \in Y$ in the form $y=q u p$, where $q \in P_{i}, u \in U_{i j}^{-}$, and $p \in P_{j}$. By the first assumption of the lemma, there exists $x \in \mathrm{EL}_{i} \cap \mathrm{EP}_{j}$ such that $q x \in \widetilde{P}$. We rewrite $y$ in the form $y=(q x)\left(x^{-1} u x\right)\left(x^{-1} p\right)$. Obviously, $x^{-1} u x \in U_{i}^{-}$, whence

$$
y \in \widetilde{P} U_{i}^{-} P_{j}=\widetilde{P} U_{i j}^{-}\left(U_{i}^{-} \cap L_{j}\right) P_{j}=\widetilde{P} U_{i j}^{-} P_{j} .
$$

This implies that $Y \subseteq \widetilde{P} U_{i j}^{-} P_{j}$. To complete the proof, it only remains to write

$$
X_{-\alpha_{i}} Y \subseteq X_{-\alpha_{i}} \widetilde{P} U_{i j}^{-} P_{j} \subseteq P_{i} \cdot \mathrm{EP}_{j}^{-} \cdot U_{i j}^{-} P_{j}=P_{i} P_{j}^{-} P_{j}=P_{i}\left(U_{j}^{-} \cap L_{i}\right) U_{i j}^{-} L_{j} P_{j}=Y .
$$

$\S 7$. The use of Stability COnditions in the CASE Where $\Phi=\mathrm{A}_{l}$

We define $\widetilde{P}$ as the set of all matrices $g \in P_{r}$ such that the row

$$
\left(g_{r, s+1}, g_{r, s+1}, \ldots, g_{r, r}\right)
$$

is unimodular. In other words, $\widetilde{P}$ is the set of all matrices $g \in P_{r}$ such that, upon writing $g$ in the block form,

$$
g=\left(\begin{array}{ccc}
g^{11} & g^{12} & g^{13} \\
g^{21} & g^{22} & g^{23} \\
0 & 0 & g^{33}
\end{array}\right),
$$

the last row of the block $g^{22}$ is unimodular.

Lemma 10. Let $\operatorname{sr}(R) \leq r-s$. Then for every $p \in P_{r}$ there exists an element $x \in$ $\mathrm{EL}_{r} \cap \mathrm{EP}_{s}$ such that $p x \in \widetilde{P}$.

Proof. Take a matrix $p \in P_{r}$ and express it as a block matrix in accordance with the partition $(s, r-s, n-r)$ :

$$
p=\left(\begin{array}{ccc}
g^{11} & g^{12} & g^{13} \\
g^{21} & g^{22} & g^{23} \\
0 & 0 & g^{33}
\end{array}\right) .
$$

Clearly, the block matrix $\left(\begin{array}{cc}g^{11} & g^{12} \\ g^{21} & g^{22}\end{array}\right)$ is invertible. In particular, its last row $\left(g_{r, 1}, \ldots, g_{r, r}\right)$ is unimodular.

Lemma 1 implies that there exists a matrix $a \in M(s, r-s, R)$ such that the row

$$
\left(g_{r, 1}, \ldots, g_{r, s}\right) a+\left(g_{r, s+1}, \ldots, g_{r, r}\right)
$$


is a unimodular row of length $r-s$. Now, we can set

$$
x=\left(\begin{array}{ccc}
e & a & 0 \\
0 & e & 0 \\
0 & 0 & e
\end{array}\right) \in \mathrm{EL}_{r} \cap U_{s} .
$$

Recall that a matrix of the form $e+u \xi v$, where $u \in R^{n}, v \in{ }^{n} R, \xi \in R$, and $v u=0$ is called a transvection. Each elementary transvection in $\mathrm{E}(n, R)$ is also a transvection in this more general sense, $t_{i j}(\xi)=e+e_{i} \xi f_{j}$. The following Whitehead type lemma is obvious and well known. Essentially, it can be traced back to Bass' paper [22], and the proof can be found, e.g., in [51, Lemma 3].

Lemma 11. Let $u \in R^{n}$ and $v \in{ }^{n} R$ be a column and a row such that $v u=0$. Assume that either $u$ or $v$ has at least one zero component. Then for any $\xi \in R$ the transvection $e+u \xi v$ belongs to $E(n, R)$.

Lemma 12. For every $p \in \widetilde{P}$ there exists an element $x \in \mathrm{EP}_{s}^{-} \cap \mathrm{EP}_{r}$ such that

$$
\left(X_{-\alpha_{r}}\right)^{p x} \subseteq \mathrm{EP}_{s} .
$$

Proof. Suppose $p \in \widetilde{P}$ and $x_{-\alpha}(\xi) \in X_{-\alpha_{r}}$. By the very definition of $\widetilde{P}$, there exist $c_{s+1}, \ldots, c_{r}$ such that

$$
\sum_{i=s+1}^{r} c_{i} p_{r, i}=1
$$

Therefore, multiplying $p$ by the corresponding element $x \in \mathrm{EP}_{s}^{-} \cap \mathrm{EP}_{r}$, we can make the entries $w_{r, 1}, \ldots, w_{r, s}$ and the entry $w_{r, l+1}$ of the matrix $w=p x$ to be zero. Now, set $u=w_{*, r+1}^{\prime}$ and $v=w_{r, *}$. To check that the matrix $x_{-\alpha_{r}}(\xi)^{w}$ lies in $\mathrm{EP}_{s}$, we express it as follows:

$$
w^{-1} x_{-\alpha_{r}}(\xi) w=e+u \xi v=(e+\tilde{u} \xi v) \prod_{i=1}^{s} \prod_{j=s+1}^{n} t_{i, j}\left(w_{i, r+1}^{\prime} \xi w_{r, j}\right)
$$

where $\widetilde{u}=u-\sum_{i=1}^{s} w_{i, r+1}^{\prime} e_{i}$. The second factor lies in $U_{s}$, and it only remains to observe that the first factor is a transvection, which lies in $\mathrm{EL}_{s}$ by Lemma 11, because

$$
\widetilde{u}_{1}=\cdots=\widetilde{u}_{s}=v_{1}=\cdots=v_{s}=v_{l+1}=0 .
$$

Proof of Theorems 1 and 2 for $\Phi=\mathrm{A}_{l}$. Let $\mathrm{EY}=\mathrm{EY}_{r s}, Y=Y_{r s}$. We want to prove that $\mathrm{EY}=E, Y=G$. Observe that for this it suffices to verify the inclusions $E \cdot \mathrm{EY} \subseteq$ $\mathrm{EY}, G \cdot Y \subseteq Y$. Next, observe that, by Lemma 6, the group $E(\Phi, R)$ is generated by $\mathrm{EP}_{r}$ and $X_{-\alpha_{r}}$, while Lemma 7 guarantees that under our assumptions on the ring $R$ the group $G(\Phi, R)$ is generated by $P_{r}$ and $X_{-\alpha_{r}}$. Thus, it only remains to verify the inclusions $g Y \subseteq Y, g \mathrm{EY} \subseteq \mathrm{EY}$ for the above generators $g$ of the groups $E(\Phi, R)$ and $G(\Phi, R)$, respectively. Since $\mathrm{EP}_{r}$ and $P_{r}$ are subgroups, it suffices to check that $X_{-\alpha_{r}} \mathrm{EY} \subseteq \mathrm{EY}$ and $X_{-\alpha_{r}} Y \subseteq Y$.

Observe that, since $x \in \mathrm{EP}_{s}^{-} \cap \mathrm{EP}_{r}$, for every $p \in \widetilde{P}$ the inclusion $\left(X_{-\alpha_{r}}\right)^{p x} \subseteq \mathrm{EP}_{s}$ established in Lemma 12 implies the identities

$$
x_{-\alpha_{r}}(\xi) p \in \mathrm{EP}_{r} \cdot \mathrm{EP}_{s} \cdot \mathrm{EP}_{s}^{-}=\left(\mathrm{EP}_{r} \cdot U_{s}\right)\left(\mathrm{EL}_{s} \cdot \mathrm{EP}_{s}^{-}\right)=\mathrm{EP}_{r} \cdot \mathrm{EP}_{s}^{-} .
$$

To conclude the proof, it only remains to apply Lemmas 7 and 8 to the subsets $\widetilde{P} \cap \mathrm{EP}_{r}$ and $\widetilde{P}$. 
$\S 8$. The use of Stability Conditions in the Cases where $\Phi=\mathrm{B}_{l}, \mathrm{C}_{l}$, And $\mathrm{D}_{l}$,

$$
\{r, s\} \neq\{l-1, l\}
$$

Below, we assume that $1 \leq s<r \leq l-1$ for $\Phi=\mathrm{B}_{l}, \mathrm{C}_{l}$. The existence of an outer automorphism switching the roots $\alpha_{l}$ and $\alpha_{l-1}$ implies that, when considering the case of $\Phi=\mathrm{D}_{l}$, we can drop the analysis of all cases where $s \leq l-2$ and $r=l-1$, assuming that $s \leq l-2, r=l$.

In the sequel it will be convenient to express an arbitrary element $g \in E(\Phi, R)$ in the block form, and to fix the notation for the corresponding blocks. For $\Phi=\mathrm{C}_{l}, \mathrm{D}_{l}$, we use the partition $(s, r-s, l-r, l-r, r-s, s)$ :

$$
g=\left(\begin{array}{cccccc}
g^{1,1} & g^{1,2} & g^{1,3} & g^{1,-3} & g^{1,-2} & g^{1,-1} \\
g^{2,1} & g^{2,2} & g^{2,3} & g^{2,-3} & g^{2,-2} & g^{2,-1} \\
g^{3,1} & g^{3,2} & g^{3,3} & g^{3,-3} & g^{3,-2} & g^{3,-1} \\
g^{-3,1} & g^{-3,2} & g^{-3,3} & g^{-3,-3} & g^{-3,-2} & g^{-3,-1} \\
g^{-2,1} & g^{-2,2} & g^{-2,3} & g^{-2,-3} & g^{-2,-2} & g^{-2,-1} \\
g^{-1,1} & g^{-1,2} & g^{-1,3} & g^{-1,-3} & g^{-1,-2} & g^{-1,-1}
\end{array}\right) .
$$

On the other hand, for $\Phi=\mathrm{B}_{l}$ all matrices will be partitioned into blocks in accordance with the partition $(s, r-s, l-r, 1, l-r, r-s, s)$ :

$$
g=\left(\begin{array}{ccccccc}
g^{1,1} & g^{1,2} & g^{1,3} & g^{1,0} & g^{1,-3} & g^{1,-2} & g^{1,-1} \\
g^{2,1} & g^{2,2} & g^{2,3} & g^{2,0} & g^{2,-3} & g^{2,-2} & g^{2,-1} \\
g^{3,1} & g^{3,2} & g^{3,3} & g^{3,0} & g^{3,-3} & g^{3,-2} & g^{3,-1} \\
g^{0,1} & g^{0,2} & g^{0,3} & g^{0,0} & g^{0,-3} & g^{0,-2} & g^{0,-1} \\
g^{-3,1} & g^{-3,2} & g^{-3,3} & g^{-3,0} & g^{-3,-3} & g^{-3,-2} & g^{-3,-1} \\
g^{-2,1} & g^{-2,2} & g^{-2,3} & g^{-2,0} & g^{-2,-3} & g^{-2,-2} & g^{-2,-1} \\
g^{-1,1} & g^{-1,2} & g^{-1,3} & g^{-1,0} & g^{-1,-3} & g^{-1,-2} & g^{-1,-1}
\end{array}\right)
$$

In what follows, all references to blocks of all occurring matrices should be understood in accordance with these partitions.

In the cases where $\Phi=\mathrm{C}_{l}, \mathrm{D}_{l}$, the elements $p \in P_{s}, q \in P_{r}$ have the form

$$
p=\left(\begin{array}{llllll}
* & * & * & * & * & * \\
0 & * & * & * & * & * \\
0 & * & * & * & * & * \\
0 & * & * & * & * & * \\
0 & * & * & * & * & * \\
0 & 0 & 0 & 0 & 0 & *
\end{array}\right), \quad q=\left(\begin{array}{cccccc}
* & * & * & * & * & * \\
* & * & * & * & * & * \\
0 & 0 & * & * & * & * \\
0 & 0 & * & * & * & * \\
0 & 0 & 0 & 0 & * & * \\
0 & 0 & 0 & 0 & * & *
\end{array}\right) .
$$

For $\Phi=\mathrm{B}_{l}$, these elements have a similar form, with additional vertical and horizontal strips of width 1 in the middle.

Obviously, when $r=l$, the above partitions become $(s, l-s, l-s, s)$ and $(s, l-s, 1$, $l-s, s)$, respectively. In other words, the horizontal and vertical strips with indices \pm 3 disappear. As an example, we reproduce the shape of the elements $p \in P_{s}, q \in P_{l}$ in the case where $\Phi=B_{l}$ :

$$
p=\left(\begin{array}{ccccc}
* & * & * & * & * \\
0 & * & * & * & * \\
0 & * & * & * & * \\
0 & * & * & * & * \\
0 & 0 & 0 & 0 & *
\end{array}\right), \quad q=\left(\begin{array}{ccccc}
* & * & * & * & * \\
* & * & * & * & * \\
0 & 0 & * & * & * \\
0 & 0 & 0 & * & * \\
0 & 0 & 0 & * & *
\end{array}\right)
$$

We construct the set $\widetilde{P}$ satisfying the assumptions of Lemma 8 . As $\widetilde{P}$ we take the set of all matrices $p \in P_{s}$ such that the row

$$
\left(p_{-s-1,-r}, p_{-s-1,-r+1}, \ldots, p_{-s-1,-s-1}\right)
$$


is unimodular. In terms of the block partitions above, $\widetilde{P}$ can be characterized as the set of all matrices in $P_{s}$ such that the last row of the block $p^{-2,-2}$ is unimodular.

Lemma 13. Assume that $R$ satisfies the stability conditions occurring in Theorem 1. Then for every $p \in P_{s}$ there exists an element $x \in \mathrm{EL}_{s} \cap \mathrm{EP}_{r}$ such that $p x \in \widetilde{P}$.

Proof. For a given matrix $p \in P_{s}$, the row

$$
\left(p_{-s-1, s+1}, \ldots, p_{-s-1,-s-1}\right)
$$

is unimodular, being the last row of its central block in the above block form of $p$.

Using the definition of the stable rank of form rings for $\Phi=\mathrm{D}_{l}$, Lemma 5 for $\Phi=\mathrm{C}_{l}$, or Lemma 4 for $\Phi=\mathrm{B}_{l}$, we can find a matrix $x \in \mathrm{EL}_{s} \cap \mathrm{EP}_{r}$ such that the last row of the block matrix $\left((p x)^{-2,-3},(p x)^{-2,-2}\right)$ is unimodular.

Now, if $r<l$, we need to additionally invoke the usual stable rank condition $\operatorname{sr}(R) \leq$ $r-s$. By Lemma 1 , there is a matrix $b \in M(l-r, r-s, R)$ such that the last row of the matrix $(p x)^{-2,-3} b+(p x)^{-2,-2}$ is unimodular. Denote by $y$ the matrix in $\mathrm{EL}_{s} \cap \mathrm{EP}_{r}$ that only differs from the identity matrix in the blocks $y^{-3,-2}$ and $y^{2,3}$, with $y^{-3,-2}=b$. It follows that the last row of the block $(p x y)^{-2,-2}$ is unimodular.

Lemma 14. We have

$$
X_{-\alpha_{s}} \widetilde{P} \subseteq P_{s} \cdot \mathrm{EP}_{r}^{-}, \quad X_{-\alpha_{s}}\left(\widetilde{P} \cap \mathrm{EP}_{s}\right) \subseteq \mathrm{EP}_{s} \cdot \mathrm{EP}_{r}^{-} .
$$

Proof. Let $p \in \widetilde{P}$, and let $x_{-\alpha_{s}}(\xi) \in X_{-\alpha_{s}}$. Denote the last row of the block $q^{-2,-2}$ by $u$. By the very definition of $\widetilde{P}$, we can find a column $c \in R^{r-s}$ such that $u c=1$. Thus, adding columns with indices $-r, \ldots,-s-1$ to the columns with indices $-s, \ldots,-1$, we can make all entries at the corresponding positions of the $-(s+1)$ st row to become zero. These additions can be interpreted as multiplication of the matrix $p$ by a certain matrix $y \in \mathrm{EL}_{r} \cap U_{s}$ on the right.

Thus, the last row of the block $(p y)^{-2,-1}$ is zero. Next, we use the Levi decomposition of the element $p y$,

$$
p y=p_{1} p_{2}, \quad p_{1} \in U_{s}, \quad p_{2} \in L_{s} .
$$

It is easily seen that the last row of the block $p_{1}^{-2,-1}$ and the first column of the block $p_{1}^{1,2}$ are zero.

Now, set $x=x_{-\alpha_{s}}(\xi) \in X_{-\alpha_{s}}$ and consider the product

$$
x(p y)=x\left(p_{1} p_{2}\right)=\left(x p_{1} x^{-1}\right) p_{2}\left(p_{2}^{-1} x p_{2}\right) .
$$

It is immediate that the first factor of the above product is contained in $\mathrm{EP}_{s}$, the second factor $p_{2}$ is contained in $P_{s}$, while the third is contained in $U_{s}^{-}$, because $U_{s}^{-} \unlhd P_{s}^{-}$. It follows that

$$
x p \in P_{s}\left(U_{s}^{-} \cdot \mathrm{EL}_{r}\right) \subseteq P_{s} \cdot \mathrm{EP}_{r}^{-} .
$$

The second inclusion is proved similarly, with the only difference that $p_{2} \in \mathrm{EL}_{s}$, which implies that $x p \in \mathrm{EP}_{s} \cdot \mathrm{EP}_{r}^{-}$. This completes the proof of the lemma.

Proof of Theorems 1 and 2 for $\Phi=\mathrm{B}_{l}, \mathrm{C}_{l}, \mathrm{D}_{l}$. Let $\mathrm{EY}=\mathrm{EY}_{s r}, Y=Y_{s r}$. By Lemma 6, the group $E(\Phi, R)$ is generated by $\mathrm{EP}_{s}$ and $X_{-\alpha_{s}}$. The assumptions on the ring $R$ in Theorem 2 imply that we can apply Lemma 7 and, consequently, $G(\Phi, R)$ is generated by $P_{s}$ and $X_{-\alpha_{s}}$.

It follows that, as in the proof for the $\mathrm{A}_{l}$ case, it only remains to verify that $X_{-\alpha_{s}} \cdot \mathrm{EY} \subseteq$ EY and $X_{-\alpha_{s}} Y \subseteq Y$, but these inclusions immediately follow from Lemmas 8, 13, and 14. 


\section{$\S 9$. The use of Stability CONDitions in the CASE \\ WHERE $\Phi=\mathrm{D}_{l}, s=l-1, r=l$}

To complete the proof of Theorems 1 and 2, it only remains to consider the case of the maximal parabolic subgroups in the group of type $\mathrm{D}_{l}$ corresponding to the last two fundamental roots. In the analysis of this case we shall write all matrices in the block form in accordance with the partition $(l-1,1,1, l-1)$. Thus, a matrix $g \in E\left(\mathrm{D}_{l}, R\right)$ is partitioned as

$$
\left(\begin{array}{cccc}
g^{1,1} & g^{1,2} & g^{1,-2} & g^{1,-1} \\
g^{2,1} & g^{2,2} & g^{2,-2} & g^{2,-1} \\
g^{-2,1} & g^{-2,2} & g^{-2,-2} & g^{-2,-1} \\
g^{-1,1} & g^{-1,2} & g^{-1,-2} & g^{-1,-1}
\end{array}\right)
$$

In this notation, the elements $p \in P_{s}, q \in P_{r}$ have the form

$$
p=\left(\begin{array}{llll}
* & * & * & * \\
0 & * & 0 & * \\
* & * & * & * \\
0 & * & 0 & *
\end{array}\right), \quad q=\left(\begin{array}{cccc}
* & * & * & * \\
* & * & * & * \\
0 & 0 & * & * \\
0 & 0 & * & *
\end{array}\right)
$$

whereas the elements $x \in L_{s} \cap P_{r}$ and $y \in L_{r} \cap P_{s}$ have the form

$$
x=\left(\begin{array}{cccc}
* & 0 & * & 0 \\
0 & * & 0 & * \\
0 & 0 & * & 0 \\
0 & 0 & 0 & *
\end{array}\right), \quad y=\left(\begin{array}{cccc}
* & * & 0 & 0 \\
0 & * & 0 & 0 \\
0 & 0 & * & * \\
0 & 0 & 0 & *
\end{array}\right) .
$$

By definition, the set $\widetilde{P}$ consists of the matrices $p \in P_{s}$ for which the entry $p_{-l,-l}$ is invertible. The proof of Theorem 1 itself in this case does not differ from that in the previous section, once we prove the analogs of Lemmas 13 and 14 .

Lemma 15. Assume that $\operatorname{sr} R \leq 1$. Then for every $p \in P_{s}$ there exists an element $x \in \mathrm{EL}_{s} \cap \mathrm{EP}_{r}$ such that $p x \in \widetilde{P}$.

Proof. Obviously, the row $\left(p_{-l, 1}, \ldots, p_{-l, l-1}, p_{-l,-l}\right)$ of length $l$ is unimodular. By assumption, there exist $c_{1}, \ldots, c_{l-1}$ such that the element

$$
p_{-l,-l}+\sum_{i=1}^{l-1} c_{i} p_{-l, i}
$$

is invertible. Setting

$$
x=\prod_{i=1}^{l-1} T_{i,-l}\left(c_{i}\right) \in \mathrm{EL}_{s} \cap \mathrm{EP}_{r},
$$

we get $p x \in \widetilde{P}$.

Lemma 16. We have

$$
X_{-\alpha_{s}} \widetilde{P} \subseteq P_{s} \cdot \mathrm{EP}_{r}^{-}, \quad X_{-\alpha_{s}}\left(\widetilde{P} \cap \mathrm{EP}_{s}\right) \subseteq \mathrm{EP}_{s} \cdot \mathrm{EP}_{r}^{-} .
$$

Proof. We fix $p \in \widetilde{P}$. By assumption, there exist $c_{-(l-1)}, \ldots, c_{-1}$ such that, for

$$
y=\prod_{i=-(l-1)}^{-1} T_{-l, i}\left(c_{i}\right) \in \mathrm{EL}_{r} \cap \mathrm{EP}_{s},
$$

we get $(q y)^{-2,-1}=0$. Now the proof can be completed exactly as that of Lemma 14 . 
At this point, the proof of Theorems 1 and 2 for this last case is completed in exactly the same way as in the previous section, with references to Lemmas 13 and 14 replaced by references to Lemmas 15 and 16.

\section{$\S 10$. Final REMARKS}

Now we state several unsolved problems ultimately related to the results of the present paper. First, let us speak of the possible generalizations.

We are positive that the present paper, together with [11, 12, 19, 20, 39], contains all ingredients necessary to solve the following problem. Obviously, this will require substantial technical efforts, to accommodate noncommutativity, nontrivial involution, and nontrivial form parameter in the calculations.

Problem 1. Prove analogs of the Bass-Kolster and the Dennis-Vaserstein decompositions for arbitrary pairs of maximal parabolic subgroups in the generalized unitary groups $U(n, R, \mathcal{L})$.

As it seems, a complete solution of the following problem will be associated with more severe technical difficulties.

Problem 2. Prove analogs of the Bass-Kolster and Dennis-Vaserstein decompositions for arbitrary pairs of maximal parabolic subgroups in the exceptional Chevalley groups.

As of today, under natural stability conditions, the Dennis-Vaserstein type decompositions for exceptional groups are only established for some pairs of terminal parabolic subgroups, and even for these cases definitive results are still not published [42. The proofs of these decompositions rely materially on the fact that the Levi subgroup of one of the parabolic subgroups in question is of type $\mathrm{A}_{l-1}$, and on the fact that the restriction of the minimal representation of the exceptional group to $G\left(\mathrm{~A}_{l-1}, R\right)$ has the vector representation of the group $\mathrm{SL}(l, R)$ as a direct summand. The preceding publications invoked the absolute stable rank, or even stronger stability conditions to establish similar results [50, 13, 14, 40, 41]; see the survey [52] for further references regarding the use of stability conditions in the theory of exceptional groups.

In reality, as we understand it today, the correct stability conditions are the higher analogs of the stable rank of form rings, stated in terms of the action of unipotent radicals of the maximal parabolic subgroups. However, for exceptional groups, most of these conditions, in particular, all conditions for parabolic subgroups with the unipotent radical of class at least 3 , are new, and were never considered before. Thus, first we need to study the conditions themselves, how they follow from the appropriate conditions on dimension, the absolute stable rank, etc.

Next, we mention some possible applications of our results. An extremely hot topic in group theory of the last decades are width estimates in terms of various systems of generators. For instance, there are dozens of papers where the width of elementary Chevalley groups in elementary generators, commutators, and the like has been estimated. However, for general rings the width in so small generators is usually infinite.

In fact, to have a fair chance to get a finite width, not to say to obtain precise bounds, one must consider terribly much larger generating sets. Below we cite two typical examples where the use of parabolic factorizations can help to calculate width, or obtain sharp estimates thereof, at least under appropriate stability conditions.

Problem 3. Let $\Delta \subseteq \Phi$. Calculate the width of $E(\Phi, R)$ in terms of $g E(\Delta, R) g^{-1}$, $g \in E(\Phi, R)$.

Problem 4. Let $\Delta \subseteq \Phi$. Calculate the width of $E(\Phi, R)$ in $w E(\Delta, R) w^{-1}, w \in W(\Phi)$. 
We also mention another possible application of parabolic factorizations, namely, to the classification of overgroups of the elementary parabolic subgroups in the style of [3, 4. This problem is closely related also to the description of overgroups of the elementary Levi subgroups inside the parabolic subgroups themselves, obtained by Anastasia Stavrova in 48 .

Problem 5. Describe the overgroups of $P(R)$ in $G(\Phi, R)$ in the case where $P$ is the (elementary) maximal parabolic subgroup of $G(\Phi, R)$.

Incidentally, it seems plausible that, with the use of representation theory, similar results can be obtained for arbitrary commutative rings, independently of stability conditions, at least when the ranks of all irreducible factors of the Levi subgroup of $P$ are not too small.

The present paper is a further elaboration of a theme that Eugene Plotkin, Mike Stein, Andrei Suslin, and the first author discussed in the Spring of 2001 in Evanstone, and in the Winter of 2002 in Ramat Gan. The main ideas of the present paper are variations of the marvelous themes invented by Mike and Andrei, and first appeared in our joint paper 42, which unfortunately remains unpublished. Eugene and the first author learned this stuff in 1976, from the preprint of [50, which Mike sent to Andrei, and from the paper 16 by Andrei and Marat Tulenbaev.

However, whereas [6] is literally an exercise on the themes of [16, 50], the present paper introduces an important new twist, which was simply not there at that time. Namely, it is the genuine stability condition, the stable rank of form rings, which appeared in the works by Anthony Bak, Victor Petrov, and Guoping Tang [19, 20, 12.

\section{REFERENCES}

[1] H. Bass, Algebraic K-theory, W. A. Benjamin, Inc., New York-Amsterdam, 1968. MR0249491 $(40: 2736)$

[2] N. Bourbaki, Lie groups and Lie algebras. Chapters 4-6, Springer-Verlag, Berlin, 2002. MR1890629 (2003a:17001)

[3] N. A. Vavilov, Subgroups of the general linear group over a ring that contains the group of block triangular matrices. II, Vestnik Leningrad. Univ. Mat. Mekh. Astronom. 1982, vyp. 3, 5-9; English transl. in Vestnik Leningrad Univ. Math. 15 (1983). MR0672588 (84g:20083)

[4] _ Parabolic subgroups of the Chevalley group over a commutative ring, Zap. Nauchn. Sem. Leningrad. Otdel. Mat. Inst. Steklov. (LOMI) 116 (1982), 20-43; English transl. in J. Soviet Math. 26 (1984), no. 3. MR 0687837 (85j:20046a)

[5] N. A. Vavilov and E. B. Plotkin, Net subgroups of Chevalley groups. II. Gaussian decomposition, Zap. Nauchn. Sem. Leningrad. Otdel. Mat. Inst. Steklov. (LOMI) 114 (1982), 62-76; English transl. in J. Soviet Math. 27 (1984), no. 4. MR0669560 (83k:20051)

[6] N. A. Vavilov and S. S. Sinchuk, Decompositions of Dennis-Vaserstein type, Zap. Nauchn. Sem. S.-Peterburg. Otdel. Mat. Inst. Steklov. (POMI) 375 (2010), 48-60; English. transl., J. Math. Sci. (N.Y.) 171 (2010), no. 3, 331-337. MR2749274 (2012a:20082)

[7] L. N. Vaserstein, On the stabilization of the general linear group over a ring, Mat. Sb. (N.S.) 79 (1969), no. 3, 405-424; English transl., Math. USSR-Sb. 8 (1969), 383-400. MR0267009 (42:1911)

[8] Stabilization of unitary and orthogonal groups over a ring with involution, Mat. Sb. (N.S.) 81 (1970), no. 3, 328-351; English transl. in Math. USSR-Sb. 10 (1970). MR0269722(42:4617)

[9] _ The stable range of rings and the dimension of topological spaces, Funktsional. Anal. i Prilozhen. 5 (1971), no. 2, 17-27; English transl., Funct. Anal. Appl. 5 (1971), 102-110. MR0284476 $(44: 1701)$

[10] , Stabilization for the classical groups over rings, Mat. Sb. (N. S.) 93 (1974), no. 2, 268-295; English transl. in Math. USSR-Sb. 22 (1974). MR0338208 (49:2974)

[11] V. A. Petrov, Odd unitary groups, Zap. Nauchn. Sem. S.-Peterburg. Otdel. Mat. Inst. Steklov. (POMI) 305 (2003), 195-225; English transl., J. Math. Sci. (N.Y.) 130 (2005), no. 3, 4752-4766. MR2033642(2005b:20100)

[12] - Overgroups of classical groups, Kand. diss., S.-Peterburg. Gos. Univ., St. Petersburg, 2005, pp. 1-129. (Russian) 
[13] E. B. Plotkin, Net subgroups of Chevalley groups and questions of stabilization of the $K_{1}$-functor, Kand. diss., Leningrad. Gos. Univ., Leningrad, 1985, pp. 1-118. (Russian)

[14] _ Surjective stabilization of the $K_{1}$-functor for some exceptional Chevalley groups, Zap. Nauchn. Sem. Leningrad. Otdel. Mat. Inst. Steklov. (LOMI) 198 (1991), 65-88; English transl., J. Soviet Math. 64 (1993), no. 1, 751-766. MR1164860(94d:19002)

[15] A. V. Stepanov, Conditions for the stability in the theory of linear groups over rings, Kand. diss., Leningrad Gos. Univ., Leningrad, 1987, pp. 1-112. (Russian)

[16] A. A. Suslin and M. S. Tulenbaev, Stabilization theorem for the Milnor $K_{2}$-functor, Zap. Nauchn. Sem. Leningrad. Otdel. Mat. Inst. Steklov. (LOMI) 64 (1976), 131-152; English transl., J. Soviet Math. 17 (1981), no. 2, 1804. MR0457525 (56:15730)

[17] E. Abe and K. Suzuki, On normal subgroups of Chevalley groups over commutative rings, Tôhoku Math. J. (2) 28 (1976), no. 2, 185-198. MR0439947 (55:12828)

[18] A. Bak, The stable structure of quadratic modules, Thesis, Columbia Univ., 1969.

[19] A. Bak, V. Petrov, and Tang Guoping, Stability for quadratic $K_{1}, K$-Theory 30 (2003), no. 1, $1-11$. MR2061845(2005c:19001)

[20] A. Bak and Tang Guoping, Stability for Hermitian $K_{1}$, J. Pure Appl. Algebra 150 (2000), 109-121. MR.1765866 (2001g:19002)

[21] A. Bak and N. Vavilov, Structure of hyperbolic unitary groups. I. Elementary subgroups, Algebra Colloq. 7 (2000), no. 2, 159-196. MR1810843 (2002b:20070)

[22] H. Bass, K-theory and stable algebra, Inst. Hautes Études Sci. Publ. Math. 22 (1964), 5-60. MR0174604 (30:4805)

[23] Unitary algebraic K-theory, Algebraic K-Theory. III: Hermitian K-Theory and Geometric Applications (Proc. Conf., Battelle Memorial Inst., Seattle, Wash., 1972), Lecture Notes in Math., vol. 343, Springer, Berlin, 1973, pp. 57-265. MR0371994(51:8211)

[24] M. Demazure and A. Grothendieck, Schémas en groupes. I, II, III, Lecture Notes in Math., vol. 151, 152, 153, Springer-Verlag, Berlin-New York, 1970. MR0274458 (43:223a) MR0274459(43:223b) MR0274460 (43:223c)

[25] R. K. Dennis, Stability for $K_{2}$, Proc. Conf. on Orders, Group Rings and Related Topics (Ohio State Univ., Columbia, Ohio, 1972), Lecture Notes in Math., vol. 353, Springer, Berlin, 1973, pp. 85-94. MR0347892(50:393)

[26] R. K. Dennis and L. N. Vaserstein, On a question of M. Newman on the number of commutators, J. Algebra 118 (1988), 150-161. MR0961333 (89k:20062)

[27] I. V. Erovenko, $S L_{N}(F[x])$ is not boundedly generated by elementary matrices: explicit proof, Electron. J. Linear Algebra 11 (2004), 162-167. MR2111520 (2005j:20034)

[28] D. R. Estes and J. Ohm, Stable range in commutative rings, J. Algebra 7 (1967), no. 3, 343-362. MR0217052 (36:147)

[29] G. Habdank, A classification of subgroups of $\Lambda$-quadratic groups normalized by relative elementary subgroups, Dissertation Universität Bielefeld, 1987, pp. 1-71.

[30] _ A classification of subgroups of $\Lambda$-quadratic groups normalized by relative elementary groups, Adv. Math. 110 (1995), no. 2, 191-233. MR1317615 (96a:20064)

[31] A. J. Hahn and O. T. O'Meara, The classical groups and K-theory, Grundlehren Math. Wiss., Bd. 291, Springer-Verlag, Berlin, 1989. MR1007302 (90i:20002)

[32] R. Hazrat and N. Vavilov, Bak's work on K-theory of rings (with an appendix by Max Karoubi), J. K-Theory 4 (2009), no. 1, 1-65. MR2538715(2011d:19001)

[33] W. van der Kallen, Injective stability for $K_{2}$, Algebraic $K$-Theory (Proc. Conf., Northwestern Univ., Evanston, IL, 1976), Lecture Notes in Math., vol. 551, Springer, Berlin, 1976, pp. 77-154. $\operatorname{MR} 0506636(58: 22243)$

[34], $\mathrm{S} L_{3}(\mathbb{C}[x])$ does not have bounded word length, Algebraic K-Theory, Part I (Oberwolfach, 1980), Lecture Notes in Math., vol. 966, Springer, Berlin-New York, 1982, pp. 357-361. MR.0689383 (84b:20037)

[35] M.-A. Knus, Quadratic and Hermitian forms over rings, Grundlehren Math. Wiss., Bd. 294, Springer-Verlag, Berlin, 1991. MR,1096299(92i:11039)

[36] M. Kolster, On injective stability for $K_{2}$, Algebraic $K$-Theory, Part I (Oberwolfach, 1980), Lecture Notes in Math., vol. 966, Springer, Berlin-New York, 1982, pp. 128-168. MR0689373 (84f:18019)

[37] B. A. Magurn, W. van der Kallen, and L. N. Vaserstein, Absolute stable rank and Witt cancellation for noncommutative rings, Invent. Math. 91 (1988), 525-542. MR0928496

[38] H. Matsumoto, Sur les sous-groupes arithmétiques des groupes semi-simples déployés, Ann. Sci. École Norm. Sup. (4) 2 (1969), 1-62. MR0240214 (39:1566)

[39] V. Petrov, Overgroups of unitary groups, K-Theory 29 (2003), 147-174. MR2028500(2004m:20094) 
[40] E. Plotkin, Stability theorems of $K_{1}$-functors for Chevalley groups, Nonassociative Algebras and Related Topics (Hiroshima, 1990), World Sci. Publ., River Edge, NJ, 1991, pp. 203-217. MR1150261 (93e:19003)

[41] On the stability of the $K_{1}$-functor for Chevalley groups of type $\mathrm{E}_{7}$, J. Algebra 210 (1998), 67-85. MR1656415 (99k:20099)

[42] E. Plotkin, M. R. Stein, and N. Vavilov, Stability of K-functors modeled on Chevalley groups, revisited, 2001 (to appear).

[43] R. W. Sharpe, On the structure of the unitary Steinberg group, Ann. of Math. (2) 96 (1972), no. 3, 444-479. MR0320076(47:8617)

[44] - On the structure of the Steinberg group $\operatorname{St}(\Lambda)$, J. Algebra 68 (1981), 453-467. MR0608545 $(82 \mathrm{k}: 20083)$

[45] J. T. Stafford, Stable structure of noncommutative Noetherian rings, J. Algebra 47 (1977), 244-267. MR0447335 (56:5648)

[46] - On the stable range of right Noetherian rings, Bull. London Math. Soc. 13 (1981), 39-41. MR.0599038 (82f:16017)

[47] - Absolute stable rank and quadratic forms over noncommutative rings, K-Theory 4 (1990), 121-130. MR 1081655 (91j:19001)

[48] A. Stavrova, Normal structure of maximal parabolic subgroups in Chevalley groups over rings, Algebra Colloq. 16 (2009), no. 4, 631-648. MR2547091 (2010j:20068)

[49] M. R. Stein, Surjective stability in dimension 0 for $K_{2}$ and related functors, Trans. Amer. Math. Soc. 178 (1973), 165-191. MR0327925 (48:6267)

[50] Stability theorems for $K_{1}, K_{2}$ and related functors modeled on Chevalley groups, Japan. J. Math. 4 (1978), no. 1, 77-108. MR0528869 (81c:20031)

[51] A. Stepanov and N. Vavilov, Decomposition of transvections: a theme with variations, $K$-Theory 19 (2000), 109-153. MR 1740757 (2000m:20076)

[52] N. A. Vavilov, A. Yu. Luzgarev, and A. V. Stepanov, Calculations in exceptional groups over rings, Zap. Nauchn. Sem. S.-Peterburg. Mat. Inst. Steklov. (POMI) 373 (2009), 48-72; English transl., J. Math. Sci. (N.Y.) 168 (2010), no. 3, 334-348. MR2749253(2012c:20136)

Department of Mathematics and Mechanics, St. Petersburg State University, UniverSitetskaya ul. 28, Stary Petergof, St. Petersburg 198504, Russia

E-mail address: nikolai-vavilov@yandex.ru

Department of Mathematics and Mechanics, St. Petersburg State University, UniverSitetskaya Ul. 28, Stary Petergof, St. Petersburg 198504, Russia

E-mail address: sinchukss@yandex.ru

Received 21/MAY/2010

Translated by THE AUTHORS 